\title{
Identification of a rapidly-spreading triple mutant for high-level metabolic insecticide resistance in Anopheles gambiae provides a real-time molecular diagnostic for anti-malarial intervention deployment.
}

Harun Njoroge ${ }^{1}$, Arjen van’t Hof ${ }^{1}$, Ambrose Oruni $^{1}$, Dimitra Pipini ${ }^{1}$, Sanjay C Nagi ${ }^{1}$, Amy Lynd $^{1}$, Eric Lucas ${ }^{1}$, Sean Tomlinson ${ }^{1}$, Xavier Grau-Bové ${ }^{1}$, Daniel McDermott ${ }^{1}$, Francis t Wat'senga $^{2}$, Emile Z Manzambi ${ }^{2}$, Fiacre R Agossa ${ }^{3}$, Arlette Mokuba ${ }^{4}$, Seth Irish ${ }^{5}$, Bilali Kabula $^{6}$, Charles Mbogo ${ }^{7}$, Joel Bargul ${ }^{8}$, Mark JI Paine ${ }^{1}$, David Weetman ${ }^{1}$, and Martin Donnelly ${ }^{1}$

${ }^{1}$ Liverpool School of Tropical Medicine

${ }^{2}$ Institut National de Recherche Biomédicale

${ }^{3}$ USAID President's Malaria Initiative

${ }^{4}$ USAID President's Malaria Initiative,

${ }^{5} \mathrm{CDC}$

${ }^{6}$ National Institute for Medical Research Amani Centre

${ }^{7}$ Kenya Medical Research Institute

${ }^{8}$ Jomo Kenyatta University of Agriculture and Technology

February 15, 2022

\begin{abstract}
Insecticide resistance provides both a pressing threat to the control of vector-borne diseases and insights into the remarkable capacity of natural populations to show rapid evolutionary responses. Malaria control remains heavily dependent on deployment of insecticides, primarily in long lasting insecticide treated nets (LLINs), but resistance in the major malaria vectors has increased over the last 15 years. Identifying genetic mechanisms causing high-level resistance in mosquitoes, which may almost entirely overcome pyrethroid efficacy, is crucial for the development and deployment of potentially resistance-breaking tools. Using the Anopheles gambiae 1000 genomes data we identified a very recent selective sweep in Ugandan mosquitoes which localized to a cluster of cytochrome P450 genes. Further interrogation revealed a haplotype involving a trio of mutations, a point mutation in Cyp6p4, an insertion of a partial Zanzibar transposable element (TE) and a duplication of the Cyp6aa1 gene. The mutations appear to have originated recently in An. gambiae from the Kenya-Uganda border region, with stepwise replacement of the double-mutant (Zanzibar TE and Cyp6p4-236M) with the triple-mutant haplotype (including Cyp6aa1 duplication), which has spread into the Democratic Republic of Congo and Tanzania. The triple-mutant haplotype is strongly associated with increased expression of genes able to metabolise pyrethroids; is strongly predictive of resistance to pyrethroids but importantly, appears less effective against LLINs co-treated with the synergist piperonyl butoxide (PBO). Frequencies of the triple-mutant haplotype remain spatially variable even within countries, suggesting an effective marker system to guide deployment decisions for limited supplies of PBO-pyrethroid co-treated LLINs across African countries.
\end{abstract}

\section{Introduction}

Insecticide resistance in disease vectors has become an influential model for understanding rapid contem- 
porary evolution but, more importantly, identifying how resistance arises and spreads is crucial for disease control. Resistance to pyrethroid insecticides in African malaria vector mosquitoes has spread to near ubiquity $[1,2]$ and, though it is often difficult to demonstrate its impact on malaria infections [3], in some cases it has reached levels that threaten the effectiveness of vector control programmes [4,5]. A better understanding of resistance distribution and mechanisms will permit a more informed selection and deployment of insecticides to combat evolving mosquito populations. Whilst our understanding of the genetic basis of insecticide resistance in mosquitoes has advanced substantially [6], especially in the important vector Anopheles funestus [7], molecular diagnostics for the major vectors in the An. gambiae complex remain limited to a handful of mutations[8] which explain a relatively small fraction of the variance in phenotype $[9,10]$ or which are now at such high frequency as to provide limited diagnostic resolution [11].

Long-lasting insecticidal nets (LLINs) are the principal tool for vector control to combat malaria, especially in sub-Saharan Africa [12]. The majority of LLINs are treated only with pyrethroid insecticides, to which resistance is now widespread [13]. Though behavioural variation and physical or physiological modifications affecting insecticide uptake may sometimes play a role, pyrethroid resistance is caused predominantly by two distinct mechanisms. The first is resistance via point mutations in the target-site of the insecticide, for pyrethroids the Voltage-gated sodium channel ( Vgsc ), which results in decreased sensitivity to the insecticide [14]; the second is metabolic resistance due to over-expression or altered activity of detoxification enzymes, of which the cytochrome P450 family is commonly considered most important $[15,16]$. Cytochrome P450 activity is inhibited by the synergist piperonyl-butoxide (PBO), and bed nets incorporating PBO are effective against P450-mediated resistance, as demonstrated by large-scale field trials $[4,17]$. Given the continued operational use of pyrethroid-containing nets, it is vital that we understand the genetic mechanisms that may impact their efficacy, to optimise bednet deployment, preferably using information from rapidly-applied DNA markers. In advance of a randomized control trial of PBO-LLINs [4], we sought to characterise pyrethroid resistance mechanisms in the primary malaria vector An. gambiae s.s. [18] in Uganda and Kenya.

The recent development of the Anopheles gambiae 1000 genomes project (Ag1000g) has led to a step change in our ability to identify DNA variation driven by selection pressure. We have been able to perform genomewide searches for regions under recent natural selection in insecticide resistant populations across Africa, and work has shown that the strongest selective sweeps in the genome are all found around genes known to be important for resistance [6, 19]. Furthermore, a whole-genome scan of copy number variants (CNVs) in the Ag1000g data revealed that increases in gene copy number were highly enriched in clusters of detoxification genes, pointing to a potentially widespread mechanism for increased gene expression [20], which, in some cases, may elevate resistance. A number of gene duplications were observed around the Cyp6aa / Cyp6p gene family cluster on chromosome $2 \mathrm{R}$, and the majority of these duplications included the gene Cyp6aa1 (Figure 1). Cyp6aa1 has been found to be overexpressed in pyrethroid resistant populations in congeneric species [21-23], but it has received very little attention compared to known insecticide-metabolizing genes such as Cyp6m2 [24, 25],Cyp6p3 [15] and Cyp9k1 [26] and its importance in resistance in An. gambiae remains unknown.

In this study, we examine a strong selective sweep detected in the Cyp6aa/Cyp6p genomic region in samples of An. gambiae s.s.from Uganda and Western Kenya. We find that the sweep is closely associated with three mutations (a SNP in Cyp6p4, a duplication of Cyp6aa1 and a partial transposable element insertion termed ZZB-TE) in tight physical and statistical linkage. The triple-mutant haplotype is associated with a high-level of pyrethroid resistance, most notably to deltamethrin. The three mutations appeared sequentially, leading to successive selective sweeps, with the triple-mutant haplotype replacing earlier variants and then spreading rapidly across East and Central Africa. We show that this haplotype is under positive selection and causes increased expression of key cytochrome P450s and through recombinant protein expression using both an $E$. coli and anSf9 -baculovirus system we show that both CYP6AA1 and CYP6P4 are capable of metabolizing pyrethroid insecticides.

\section{Methods}

Interrogation of the Ag1000g dataset and identification of tagging markers 
Whole genome sequence data in the Ag1000g data set have previously revealed a strong selective sweep in Ugandan populations around the Cyp6aa/Cyp6p cluster [19] (Figure 1). An isoleucine to methionine substitution in codon 236 of CYP6P4 (see extended data Figure 10b in [19]) was identified provisionally as a swept haplotype tagging SNP. Previous work [20] had shown that a duplication of theCyp6aa1 gene was also observed in these samples (previously termed Cyp6aap-Dup1). To objectively determine how these mutations segregated with the observed selective sweep [19] we grouped the 206 Ugandan haplotypes $(n=103$ diploid individuals) by similarity using the 1000 SNPs located immediately upstream and downstream of the start of the Cyp6aa/Cyp6p gene cluster (500 non-singleton SNPs in each direction from position 2R:28,480,576). Distances were calculated with the pairwise_distance function inscikit-allel [27] and converted to a nucleotide divergence matrix (Dxy statistic) by correcting the distance by the number of sequencing-accessible bases in that region. We defined clusters of highly similar haplotypes by hierarchical clustering with a cutoff distance of 0.001. This resulted in the identification of a cluster of 122 highly similar haplotypes. To determine whether the haplotype cluster showed signs of a selective sweep, we estimated the extended haplotype homozygosity (EHH) decay within each of the haplotype groupings, around two different focal loci: (i) the putative sweep SNP marker Cyp6p4-236M (2R:28,497,967 +/- $200 \mathrm{kbp}$; total 14,243 phased variants), and (ii) the 5' and 3' breakpoints of the Cyp6aap-Dup1 duplication (2R:28,480,189 - $200 \mathrm{kbp}$ and 2R:28,483,475 + $200 \mathrm{kbp}$; total 14,398 phased variants). We used theehh_decay function of scikit-allel[27].

We also used the haplotype groupings identified above to calculate the Garud $H$ statistics [28] and the haplotypic diversity at the Cyp6aa/Cyp6p cluster locus (coordinates 2R:28,480,576 to 2R:28,505,816). Specifically, we used themoving_garud_h and moving_haplotype_diversityfunctions in scikit-allel to obtain a series of estimates for each statistic in blocks of 100 variants located within the cluster, and used a block-jackknife procedure to calculate averages and standard errors of each estimate (jackknife function inscikit-allel misc module).

\section{Molecular screening of colony and wild caught mosquitoes}

Locked-nucleic acid (LNA) probe-based PCR diagnostics were designed for all three mutations (Supplementary materials Appendix 1).

Genotype:phenotype association testing was performed using two colonies of An. gambiae s.s., BusiaUG (resistant) and Mbita (susceptible). The BusiaUG strain was established in the lab in November 2018 from Busia, eastern Uganda and exhibits high resistance to pyrethroids and organochlorines $(<10 \%$ mortality following WHO exposure test), but full susceptibility to organophosphates and carbamates (Oruniet al. unpublished). The Mbita strain was first colonised from Mbita Point, Kenya in 1999, and is fully susceptible to pyrethroids. Colonies were reared in insectaries targeted to $25-27^{\circ} \mathrm{C}$ and $70-80 \%$ relative humidity.

Freshly emerged females from the Mbita line were mated with 3-5 day-old BusiaUG males and then blood fed. The offspring from this cross were then crossed back to the parental BusiaUG line. This design was chosen as resistance variants are often recessive. The resultant backcrossed 3-5-day-old females were exposed for one hour to deltamethrin, permethrin or $\alpha$-cypermethrin (the three insecticides most commonly used on LLINs) or DDT (a non-pyrethroid sodium channel antagonist), following WHO standard procedures. Mosquitoes were maintained on a $10 \%$ sugar solution after exposure and mortality was recorded 24 hours post exposure.

Democratic Republic of Congo. Anopheles gambiae s.s. were obtained from the President's Malaria Initiative supported entomological surveillance project [29] and from collections conducted by Lyndet al [30]. Mosquitoes were collected by human landing catch or pyrethrum spray collection from 15 locations between 2013 and 2018. Resistance-phenotyped individuals were also obtained from Pwamba, Bassa and Fiwa in Nord Ubangi Province in 2016. These mosquitoes were assessed for susceptibility to deltamethrin or permethrin using a standard WHO tube assay or a cone bioassay where Permanet 3.0 (deltamethrin plus PBO) or Olyset Plus (permethrin plus PBO) were the test nets [23]. Kenya and Uganda. Collection details for specimens from contiguous areas of western Kenya and eastern Uganda have been published previously [20, 24, 25].Tanzania. Mosquito collections were conducted in Geita, Bagamoyo and Muleba districts of Tanzania 
in 2018 .

Mosquitoes from all collections were genotyped at the ZZB-TE,Cyp6p4-236M and Cyp6aap-Dup1 loci and genotype:phenotype association testing was performed by Fisher's exact tests.

\section{Analysis of temporal change}

The most complete time series of ZZB-TE, Cyp6p4-236M and Cyp6aap-Dup1 allele frequencies were available from Kabondo, DRC; western Kenya and eastern Uganda. To model allele frequency changes over time we estimated the three parameters of the standard recursive population genetic model (allele frequency at time zero, selection coefficient and dominance coefficient) using a maximum likelihood approach assuming a binomial distribution: an approach previously applied to insecticide target-site resistance mutations [31]. The analysis was performed in R (http://www.r-project.org). An estimated generation time of one calendar month was used as in previous studies [31].

\section{Recombinant protein and insecticide metabolism}

The CYP6P4-236M variant, and its redox partner cytochrome P450 reductase gene (CPR), were expressed in E. coli as per standard protocols [32] (Supplementary materials Appendix 2). Initial efforts to generate recombinant $C Y P 6 A A 1$ in an $E$. coli system with optimised codon usage failed, and we therefore used an Sf9-baculovirus-based expression system. Since P450 catalytic activity is dependent on electrons supplied by $\mathrm{NADPH}$ via CPR, insecticide metabolism was assayed with cell pellets of CYP6P4/CPR or CYP6AA1/CPR in the presence or absence of NADPH. The depletion of the substrate and the appearance of metabolites were monitored by reverse-phase HPLC (Supplementary materials Appendix 3).

\section{Estimation of gene expression of key P450s in triple mutant haplotype}

To determine whether the presence of the triple mutant haplotype type was associated with differential expression of genes, we examined individual females from the BusiaUg colony (Triple mutant Freq. 0.297; 95\% CI 0.233-0.370). Two legs were removed from individual mosquitoes for DNA analysis with the remaining mosquito kept for RNA analysis. DNA was extracted from legs by boiling in STE buffer at $95^{\circ} \mathrm{C}$ for 90 minutes and individuals were genotyped using the LNA qPCR assays. RNA was then extracted individually from 8 mosquitoes in each genotypic group - homozygotes for the triple mutant haplotype, wild-type homozygotes, and heterozygotes, using the Arcturus Picopure RNA isolation kit (Thermofisher). We then performed SYBR green based qPCR to measure the expression of Cyp6aa1 and Cyp6p 4 together with the known resistancelinked variant Cyp6p3 using the housekeeping genes 40s ribosomal protein S7 (AGAP010592) and elongation factor $\mathrm{Tu}$ (AGAP005128) for normalisation. The $\Delta \Delta \mathrm{CT}$ values were tested for normality and homogeneity of variances using the Shapiro-Wilks test, and the Bartlett test, respectively. A significant difference in gene expression between the genotypic groups was determined by a two-tailed two-sample Student's t-test on $\Delta \Delta \mathrm{CT}$ values, with a threshold of $\mathrm{P}=0.05$.

\section{Results}

Hierarchical clustering of $206 \mathrm{Ag} 1000 \mathrm{~g}$ haplotypes from An. gambiae s.s. from Uganda resulted in identification of a cluster of 122 haplotypes around the Cyp6aa / Cyp6p gene cluster, putatively representing a swept haplotype in this region. To characterise the signatures of selection in Ugandan haplotypes around this cluster, we examined the profile of extended haplotype homozygosity around the position of the Cyp6p4236M SNP and around the CNV inCyp6aa1. In both cases, we found that the putative swept haplotype had longer stretches of homozygosity than wild-type haplotypes (Figure 2). In addition, we found that $A n$. gambiae s.s. from Uganda had reduced haplotypic diversity along the entireCyp6aa/Cyp6p gene cluster $(h$ $=0.339746+/-0.005664$ standard error) and a combination of Garud's $H$ statistics that was indicative of a hard selective sweep in this region $\left(\right.$ high $H_{12}=0.821867+/-0.006308 \mathrm{SE} ;$ low $H_{2} / H_{1}=0.016779+/-0.000228$ $\mathrm{SE}$ [28]. These results confirm that the haplotypes we have identified have undergone a selective sweep. We then used iterative read mapping of individuals homozygous for the sweep to search for additional mutations that might be distinctive of the haplotype. This revealed that a partial copy of a Ty3/Gypsy Zanzibar 
transposon insertion (termed ZZB-TE), lacking functional open reading frames, was linked to Cyp6p4-236M and Cyp6aap-Dup1 (Figure 2).

\section{The evolution of the ZZB-TE, Cyp6p4-236M andCyp6aap-Dup1 haplotypes}

Based upon Ag1000g data and a time series of collections from Central and East Africa, we were able to trace the sequence of mutational events (ZZB-TE, Cyp6p4-236M and Cyp6aap-Dup1 ) and reconstruct the evolutionary history of the swept haplotype. Among the Ag1000g data [19], the Cyp6p4-236M mutation was only observed in collections from eastern Uganda (collected in 2012) suggesting that this mutation originated in the eastern Ugandan/western Kenyan region. In a screen of collections from Uganda and Kenya predating the Ag1000g collections by eight years (2004) (Fig 3) only the ZZB-TE insertion was detected, although the sample size was too small $(\mathrm{n}=4)$ to conclude that the Cyp6p4-236M allele was absent. The Cyp6p4-236M mutation was first observed in this region in 2005 (frequency Cyp6p4-236M =0.10) in individuals carrying the ZZB-TE mutation, whilst the Cyp6aap-Dup1 CNV was first recorded in 2008 (proportion of individuals with Dup1 $=0.8 \%$ ). This inferred sequence of events may explain why ZZB-TE and Cyp6p4-236M mutations are in tighter statistical linkage with each other than withCyp6aap-Dup1 (Figure 2), despite the closer proximity of ZZB-TE and Cyp6aap-Dup1 (Figure 1). Given the very tight association between the ZZB-TE insertion and the Cyp6p4-236M SNP, we will henceforth refer to the Cyp6p4-236M (double mutant) haplotype and the Cyp6aap-Dup1 (triple mutant). The double mutant haplotype shows a steady increase in frequency between 2004 and 2011 in Kenya (Figure 3); possibly in response to the introduction and subsequent intensification of bednet distribution programmes [29, 33, 34]. Following its appearance in 2008, the triple mutant haplotype, rapidly increased towards fixation in both collections from Uganda and Kenya, replacing the double mutant. This haplotype replacement and the observation that the triple mutant is the only non-wildtype haplotype observed outside Kenya/Uganda (such as in Tanzania and DRC, Figure 1 and 3) strongly implies an additional selective advantage to the triple mutant. The time series data from across DRC are particularly striking both in terms of the speed of increase of the triple mutant but also the north-south heterogeneity, with very low frequencies in the more southerly provinces (Figure 3).

\section{Identifying potential drivers of haplotype frequency increase}

The genomic region into which the ZZB-TE inserted does not show histone signals of regulatory activation (H3K27ac, H3K9ac) or repression (H3K9me3), and ATAC-seq data suggests it is not in an open chromatin region [35]. However we took two in silico approaches to determine whether the ZZB-TE insertion (748bp) carried putative regulatory variants. The inserted region had $98.5 \%$ sequence identity to a putative enhancer (2R:45966598-45966822) identified by homology withDrosophila melanogaster [35]. However, despite the similarity, the insertion lacked enhancer-like combination of chromatin marks identified in [35] and its potential regulatory role in nearby genes is unclear. The second approach involved screening the ZZB-TE inserted sequence for putative enhancers using iEnhancer-2L[36] and iEnhancer-EL [37]. In a windowed analysis of 200bp with a 1bp step across the entire length of ZZB-TE both predicted that some of the windows would have strong enhancer activity, however the windows were not-concordant, precluding further analysis.

Given that cytochrome P450 mediated resistance is commonly associated with differential gene expression we performed transcription studies within the Cyp6aа/Сyp6p cluster between the most contrasting haplotypes, wild-type and triple mutant, present in the BusiaUg colony. The group homozygous for the triple mutant haplotype significantly overexpressed both Cyp6aa1 (2.23-fold, 95\% CI: 1.73-2.90, P=0.0003) and Cyp6p 4 (2.57-fold, $95 \%$ CI 1.25-5.93, $\mathrm{P}=0.039)$ compared to wild-type individuals. The ratio of expression of Cyp6aa 1 broadly reflected the expected pattern based on genotype (ie 2:1.5:1 for triple mutant homozygotes: heterozygotes: wild type genotypes, respectively. Figure 4). As a control we examined a neighbouring, very commonly resistance-associated gene, Сyp6p3, but triple mutant and wild type homozygotes did not differ significantly in expression (1.33 fold, $95 \%$ CI 0.64-2.74, P $>0.05$ ).

To investigate whether resistance may be driven at least in part by an effect of the allelic variant on metabolic activity of CYP6P4, we expressed the wild-type (236I) and mutant (236M) forms in an E. coli based recom- 
binant protein system (Supplementary materials Appendices 2 and 4). Both alleles were shown to be capable of metabolizing class I (permethrin) and II (deltamethrin) pyrethroids but there was no evidence that the mutant (236M) or wildtype (236I) alleles had different rates of pyrethroid depletion. We also expressed the duplicated P450 CYP6AA1, in an Sf9 -baculovirus protein expression system. Again, metabolism assays demonstrated that the enzyme was capable of metabolizing both deltamethrin and permethrin (Supplementary materials Appendices 3 and 4 ). Depletion of deltamethrin was $36.6 \%$ greater $(\mathrm{SE}=3.79$ ) in the presence of $\mathrm{NADPH}$ than in the control ( $\mathrm{t}$-test: $\mathrm{t}=-9.67$; d.f. $=8 ; \mathrm{P}=9.6 \times 10^{-6}$ ), demonstrating that CYP6AA1/CPR is capable of metabolizing deltamethrin in vitro . Similarly, permethrin was metabolised by CYP6AA1/CPR, with permethrin being depleted by $22.4 \%$ ( $\mathrm{SE}=0.63$ ) compared to the control without NADPH ( $\mathrm{t}$-test: $\mathrm{t}=$ -31.08 ; d.f. $\left.=14 ; \mathrm{P}=2.55 \times 10^{-14}\right)$.

Given clear evidence of increased expression of both Cyp6aa1 andCyp6p4 in the triple mutant haplotype and the ability of both enzymes to metabolise pyrethroids in vitro, we investigated whether the mutations were significantly associated with resistancein vivo. Exposure of An. gambiae females from Busia, Uganda and Nord Ubangi, DRC to new LLINs in cone assays resulted in negligible mortality to the pyrethroid only LLINs, Olyset and Permanent 2.0 (Figure 5). Simultaneous exposure to pyrethroid plus the P450 inhibitor PBO in Olyset + and the top of Permanent 3.0 nets resulted in a marked reduction in resistance, demonstrating that the resistance phenotype is substantially mediated by P450s. We performed laboratory backcrosses between additional mosquitoes from Busia with the pyrethroid susceptible Mbita colony, and found that the triple mutant haplotype was significantly associated with resistance to the most commonly used type II pyrethroids in LLINs: deltamethrin (Fisher's exact test $\mathrm{p}=3.2 \times 10^{-6}$ ) and alphacypermethrin (Fisher's exact test $\mathrm{p}=5.9 \times 10^{-7}$ ) resistance although not to permethrin (Fisher's exact test $\mathrm{p}=0.06$ ) nor, as a control, DDT (Fisher's exact test $\mathrm{p}=0.84$ ) (Table 1) in WHO tube assays. Similarly, specimens collected in 2016 from the DRC showed a strong association between the triple mutant genotype and survival rate 24 hours post-exposure to either $0.05 \%$ deltamethrin for 1 hour or 3 -minute exposures to deltamethrin-treated sides of a new PermaNet 3.0 net (Table 2). No association was found in samples exposed to permethrin (24 hour WHO tube assay) or permethrin-treated Olyset Plus nets (3-minute WHO cone assay) (Table 2). Complete linkage of the three mutants in the BusiaUG colony and the DRC wild caught collections precludes determination of the relative contribution of each of the three mutations to the resistance phenotype but taken together these results demonstrate a strong impact of the triple mutant on the efficacy of pyrethroid resistance.

\section{Discussion}

We have identified a sequential series of fitness-augmenting mutations in An. gambiae culminating in a triple-mutant haplotype with a large effect on pyrethroid resistance and which is spreading rapidly across East and Central Africa. The mutation that is probably the oldest in this series, the insertion of ZZB-TE, was first detected in 2004 in the malaria-endemic area around Lake Victoria, with theCyp6p4-236M SNP evident in 2005 samples and the third, a duplication in Cyp6aa1 detected in 2008. In samples collected only five years later, the triple mutant was detected hundreds of kilometres away in the DRC. These patterns suggest both a large fitness advantage arising from the triple mutant, and a frightening speed at which resistance-conferring mutations are able to spread within and across populations.

Second generation nets treated with the synergist PBO were shown to be much more effective than conventional nets in killing mosquitoes in populations where the triple mutant haplotype is present (Figure 5). Therefore the use of PBO bednets should be prioritised in the regions where this mutation is present. A strong corollary of this finding comes from the cluster randomised control trial conducted in Uganda, where the mutation is at high frequency, which demonstrated that malaria parasite prevalence in children $<10$ years old $(12 \%$ vs $14 \%$; Prevalence ratio $=0.84,95 \%$ CI $0.72-0.98 ; \mathrm{P}=0.029)$ and mean number of mosquitoes (Density ratio $=0 \cdot 25,95 \%$ CI $0 \cdot 18-0 \cdot 3 ; \mathrm{P}<0 \cdot 0001$ ) per house were significantly lower in villages that had received PBO LLINs relative to standard LLINs [4]. There is some evidence that the haplotype may be less strongly associated with resistance to permethrin than deltamethrin (and perhaps also alphacypermethrin), although both pyrethroids were metabolised by Cyp6aa1 and Cyp6p 4 . 
Our results highlight the importance of gene duplications for the evolution of insecticide resistance. In An. gambiae, duplications have recently been shown to be concentrated in regions associated with metabolic resistance, and over 40 such duplications have been described across the genome [20]. Thirteen different duplications have so far been described that encompass Cyp6aa1 (Figure 1), both in West and East Africa and in the two sister-species An. gambiae ss and An. coluzzii [20]. It seems likely that these other Cyp6aa1duplications are also associated with pyrethroid resistance. For example, in An. coluzzii sampled from a highly insecticide-resistant population from Cote d'Ivoire, greater than $95 \%$ of individuals had Cyp6aap duplications (Supplementary materials Appendix 5). Five different duplications were observed in the collections from 2012 and 2017 and, whilst we detected no association with pyrethroid resistance, two duplications (Cyp6aap-Dup7 and Cyp6aap-Dup14) showed significant increases in frequency over time. Moreover the total number of CNVs per sample (measured as presence of each of the 5 duplications, summed for each sample) increased significantly from an average of 1.59/ individual in 2012 to 2.08 in 2017 (Mann Whitney $\mathrm{U}$ test; $\mathrm{P}<0.0001$ ); the mean number of duplications $>2$ indicates that there are multiple CNVs on the same haplotype. Duplications of the Cyp6aa1 orthologue have been found in another important malaria vector, An. funestus, from west and central Africa [21]. The Cyp6aa1 orthologue inAn. funestus, which shares an $87 \%$ identity with An. gambiae, was also observed to metabolize permethrin and deltamethrin and, when expressed in transformed Drosophila, was associated with significant increases in resistance to both permethrin and deltamethrin relative to control mosquitoes [21]. This evolution of multiple Cyp6aa1 duplications suggests this is an important Africa wide resistance mechanism

There are now several cases of insecticide resistance evolution where an initial mutation in a genomic region is followed by the spread of additional mutations on the resistant haplotype background [14, 38-42]. It is not yet clear whether the sequence of mutations that we have identified rely on each-other for effect, and thus could only have spread sequentially, or whether each additional mutation coincidentally appeared on the background of an already common mutant haplotype. In the case of Cyp6p4-236M and Cyp6aap-Dup1 it seems that the latter is more likely, although we cannot exclude the possibility that the duplication affects the regulation of Cyp $6 p 4$. In contrast, the putative enhancer inserted with the ZZB transposon may affect either or both of Cyp6p4 and Cyp6aa1, and may thus interact with the other two mutations in ways that have yet to be determined. Transposable elements can sometimes affect gene expression of neighbouring genes [38] and are abundant in mosquito genomes [43, 44]. Interestingly, in the common house mosquito, Culex pipiens , TEs have been found in the flanking regions of theEster locus, a genomic region in which many independent gene duplications have arisen and spread worldwide in response to selection from organophosphates [45]. Clearly, both TEs and gene duplications are an understudied, yet common source of variation that may have important implications for vector control efforts. The appearance and rapid spread of the three mutations described here is broadly coincident with the scale up of LLIN coverage in DRC, Kenya and Uganda. The haplotype is a strongly predictive marker of high-level resistance to pyrethroids, is easily screened with a single diagnostic assay and we suggest should be used for both insecticide resistance monitoring strategies and for informing LLIN selection.[6, 29, 46].

\section{Acknowledgements}

This work was supported by the National Institute of Allergy and Infectious Diseases ([NIAID] R01AI116811) with additional support from the Medical Research Council (MR/P02520X/1). The latter grant is a UK-funded award and is part of the EDCTP2 programme supported by the European Union. HN and AO were supported by Wellcome Trust MSc Training Fellowships in Public Health and Tropical Medicine. SI is funded by the U.S. President's Malaria Initiative. CM was supported by the Bill and Melinda Gates Foundation through the WHO (Award \#54497) and by the Biovision Foundation (Grant No.BV HH-07). JLB is supported by DELTAS Africa Initiative grant \# DEL-15-011 to THRiVE-2. The DELTAS Africa Initiative is an independent funding scheme of the African Academy of Sciences (AAS)'s Alliance for Accelerating Excellence in Science in Africa (AESA) and supported by the New Partnership for Africa's Development Planning and Coordinating Agency (NEPAD Agency) with funding from the Welcome Trust grant \# 107742/Z/15/Z and the UK government. SI is funded by the U.S. President's Malaria Initiative. The findings and conclusions in this paper are those of the authors and do not necessarily represent the 
official position of CDC, the NIAID, the National Institutes of Health or the other donors.

\section{Author contributions}

HN, AvH, MJIP, DW and MJD designed the study; HN, AvT, AO, DP, SCN, AL conducted lab and insectary experiments; HN, AvH, SCN, AL, ERL, ST, XG-B, DM, MJD performed analysis; AO, AL FW, EM, FA, SI, BK, DW, CM conducted field collections and phenotyping; HN,ERL, DW, MJD wrote the manuscript with input from all authors; JLB, CM, MJIP, DW and MJD supervised the study; all authors approved the final version of the manuscript.

\section{Data Availability Statement}

Sequence read alignments and variant calls from Ag1000G are available from the European Nucleotide Archive under study accessions PRJEB18691 and PRJEB36277 (ENA http://www.ebi.ac.uk/ena ). Python scripts to reproduce all analyses detailed in this manuscript are available athttps://github.com/xgrau/cyp6AgUganda

\section{Tables and figures}
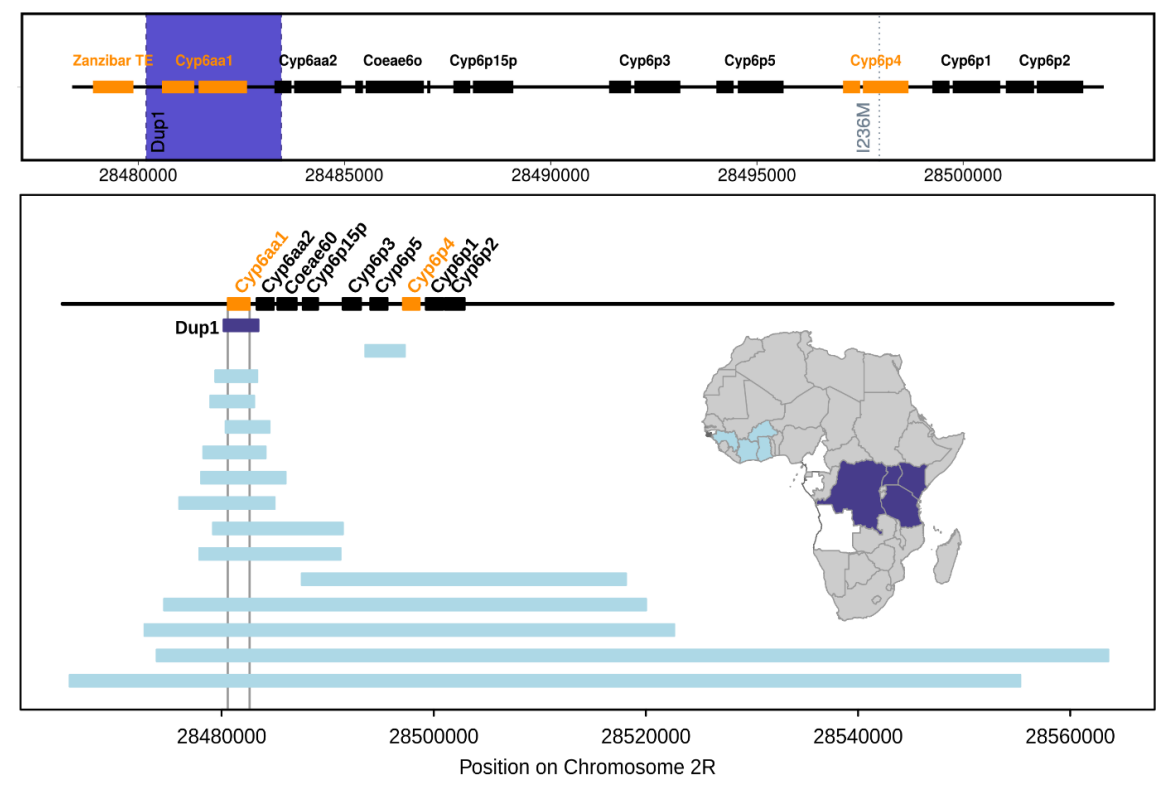

Figure 1. Schematic of the mutational events around the Cyp6aa/Cyp6p cluster on chromosome 2R in Anopheles gambiae. Upper panel shows the three mutational events observed in East and Central African samples. Orange indicates the genes/ transposable elements involved and the blue band the extent of the Cyp6aap-Dup1 CNV. The lower panel, redrawn from [20], shows the genomic extent and geographic distribution of the CNVs that have been observed around the Cyp6aa/Cyp6p cluster, 13 out of 15 include Cyp6aa1.The dark blue shading on the map indicates the geographic extent of Cyp6aap-Dup 1. Data from Uganda, Kenya and DRC are reported extensively in the main text, in addition 27.4\% ( $\mathrm{n}=84$ ) of Anopheles gambiae females sampled from northern Tanzania in 2018 were triple mutant carriers.

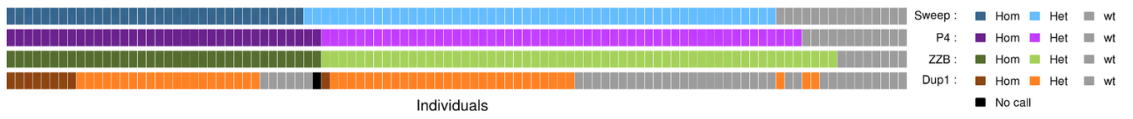



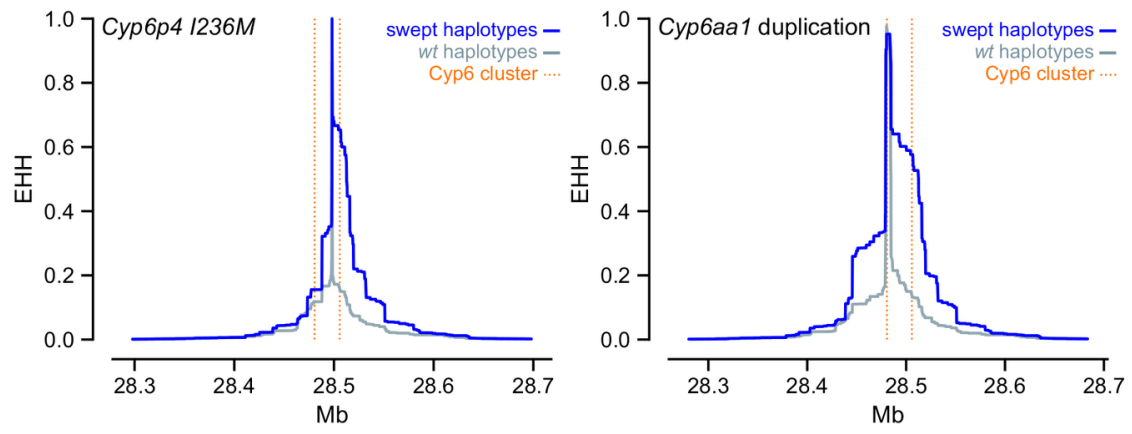

Figure 2. Selective sweep around the Cyp6aa/Cyp6p cluster inAnopheles gambiae from Uganda.

Upper panel Haplotype contingency for the selective sweep ("Sweep"),Cyp6p4-236M SNP ("P4") and the Cyp6aap-Dup1 ("Dup1"). Each vertical bar represents a single haplotype, colour-coded to show whether it is a copy of the swept haplotype (blue) and whether it carries the SNP (purple), the TE insertion (green) and the duplication (orange). The swept haplotype and the Cyp6p4-236M SNP overlap almost completely, while the duplication is found on a subset of these haplotypes. Lower panel Extended Haplotype Homozygosity (EHH) plots around the CYP6P4-236M and CYP6AA1-Dup1 variants show slower loss of homozygosity in the swept haplotypes than in the wild-type.
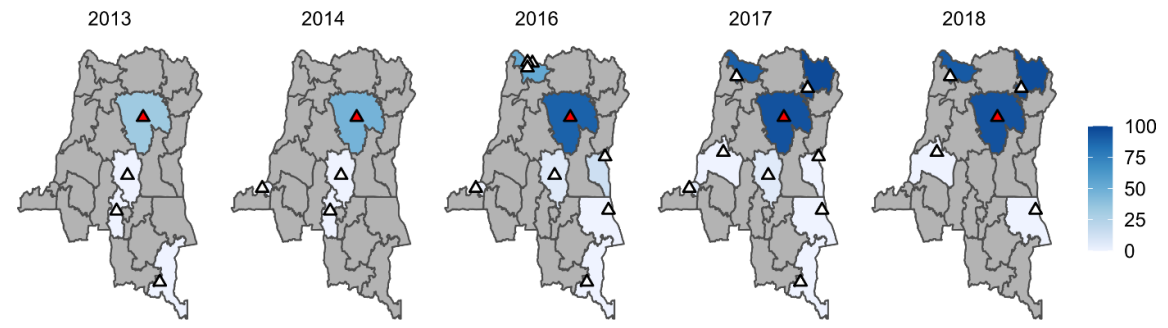

Figure 3. Observed and modelled changes in Cyp6aa/Cyp6phaplotype frequencies over time in Anopheles gambiae s.s.populations from DRC, Kenya and Uganda. Upper panel shows mutation frequency estimates derived from wild caught individuals from Kabondo, DRC; western Kenya and eastern Uganda, The 95\% CIs for each observed data point were calculated according to[47]. Expected data generated from simultaneous maximum likelihood estimates of initial frequency and selection and dominance coefficients which were then used to parameterize standard recursive allele frequency change equation. The lower panel shows data from DRC tracking the emergence and spread of the triple mutant haplotype. Triangles indicate collection locations within each district. The red triangle shows the Kabondo sample site for which there was the most complete time series. 

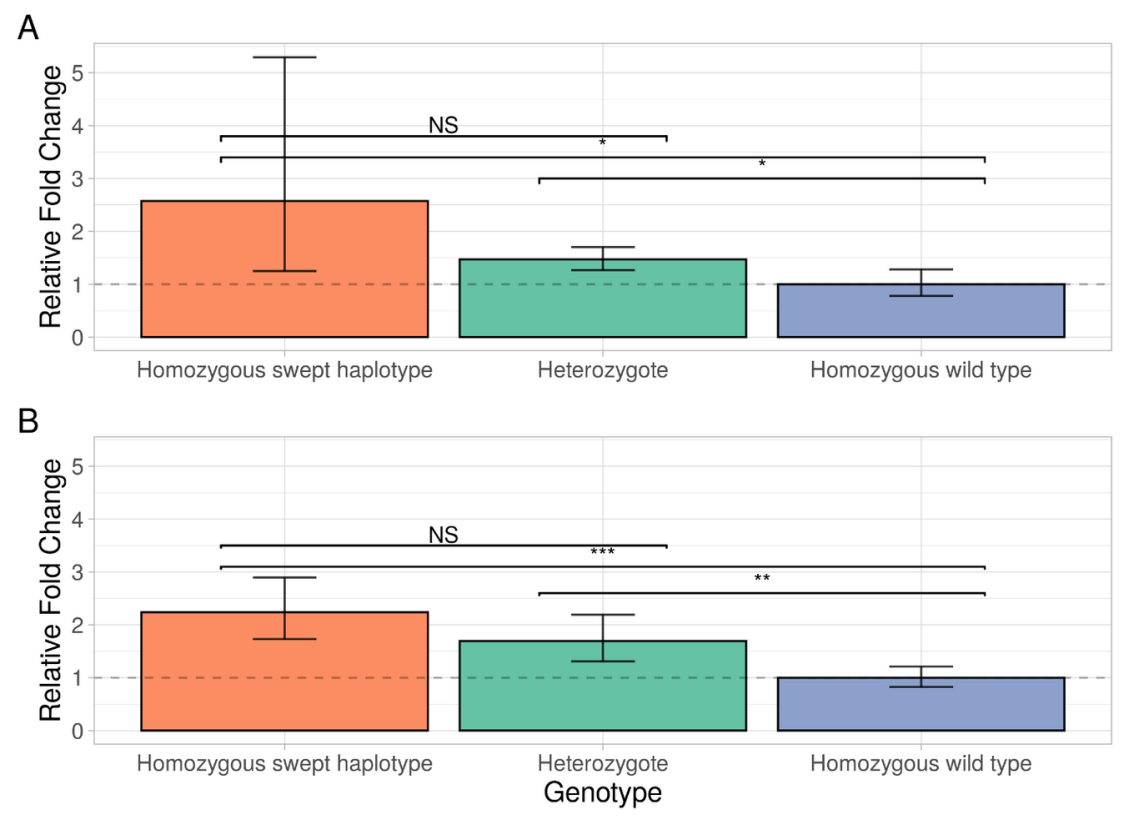

Figure 4. Gene expression analysis of Cyp6p4 and Cyp6aa1 in genotyped Anopheles gambiae females. Relative fold change for Cyp6p4 (Panel A) and Cyp6aa1 (Panel B), comparing individuals homozygous for the swept triple mutant haplotype and heterozygotes in the BusiaUG colony to wild-type individuals. 95\% confidence intervals are shown. Asterisks indicate statistical significance in two-tailed students t-tests; *** P [?] 0.001, ** P [?] 0.01, ** P [?] 0.05 .

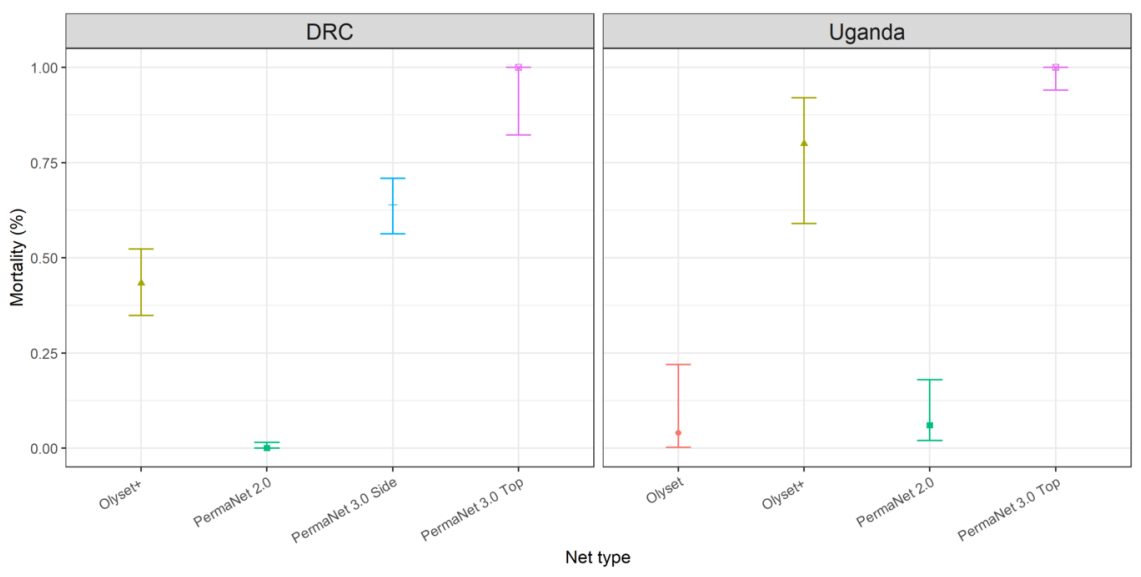

Figure 5. Patterns of insecticide resistance in Anopheles gambiae following exposure to permethrin and deltamethrin treated nets. Female Anopheles gambiae s.s. from the Democratic Republic of Congo (DRC) and Uganda exhibited very high resistance (low \% mortality) in WHO cone assays to permethrin (Olyset) and deltamethrin (PermaNet 2.0) LLINs. There was an increase in mortality following exposure to an increased concentration of deltamethrin (PermaNet 3.0 Side) and very high mortality following exposure to pyrethroid plus the P450 inhibitor piperonyl butoxide (PBO) Olyset+ and PermaNet 3.0 Top. $95 \%$ confidence intervals are shown.

Table 1. Association between insecticide susceptibility as determined by WHO tube bioassay 
and triple mutant genotype in femaleAnopheles gambiae from a cross between BusiaUG and

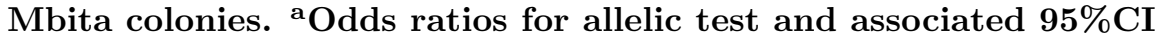

\begin{tabular}{|c|c|c|c|c|c|}
\hline Insecticide & Phenotype & $\begin{array}{l}\text { Wildtype } \\
\text { homozygote }\end{array}$ & Heterozygote & $\begin{array}{l}\text { Triple mutant } \\
\text { homozygote }\end{array}$ & $\begin{array}{l}\text { Test and } p \\
\text { value }\end{array}$ \\
\hline $\begin{array}{l}\alpha- \\
" \psi \pi \varepsilon \rho \mu \varepsilon \tau \eta \rho \nu\end{array}$ & Alive & 1 & 32 & 24 & $\begin{array}{l}\text { Genotypic } \\
\mathrm{p}=5.92 \times 10^{-7}\end{array}$ \\
\hline $\mathrm{n}=146$ & Dead & 14 & 68 & 7 & $\begin{array}{l}\text { Allelic } \\
\mathrm{p}=6.34 \times 10^{-5} \\
\mathrm{OR}^{\mathrm{a}} 2.74 \\
(1.63-4.69)\end{array}$ \\
\hline Deltamethrin & Alive & 0 & 0 & 8 & $\begin{array}{l}\text { Genotypic } \\
\mathrm{p}=3.21 \times 10^{-6}\end{array}$ \\
\hline $\mathrm{n}=60$ & Dead & 15 & 26 & 11 & $\begin{array}{l}\text { Allelic- } \\
\text { Cannot be } \\
\text { calculated }\end{array}$ \\
\hline Permethrin & Alive & 1 & 18 & 7 & $\begin{array}{l}\text { Genotypic } \\
\mathrm{p}=0.059\end{array}$ \\
\hline $\mathrm{n}=53$ & Dead & 5 & 20 & 2 & $\begin{array}{l}\text { Allelic p=0.08; } \\
\text { OR 1.99 } \\
(0.86-4.67)\end{array}$ \\
\hline DDT & Alive & 0 & 14 & 1 & $\begin{array}{l}\text { Genotypic } \\
p=1\end{array}$ \\
\hline$n=65$ & Dead & 3 & 45 & 2 & $\begin{array}{l}\text { Allelic } \mathrm{p}=0.84 \\
\text { OR 1.19 } \\
(0.48-2.94)\end{array}$ \\
\hline
\end{tabular}

Table 2. Association between insecticide susceptibility as determined by WHO tube bioassay or WHO net bioassay and triple mutant genotype in wild-caught, female Anopheles gambiae from three locations in Nord Ubangi Province, Democratic Republic of Congo. ${ }^{a}$ Odds ratios for allelic test and associated $95 \% \mathrm{CI}$

\begin{tabular}{|c|c|c|c|c|c|c|c|}
\hline Site & Insecticide & Bioassay & Phenotype & $\begin{array}{l}\text { Wildtype } \\
\text { homozygote }\end{array}$ & Heterozygote & $\begin{array}{l}\text { Triple } \\
\text { mutant } \\
\text { homozygote }\end{array}$ & $\begin{array}{l}\text { Test and } p \\
\text { value }\end{array}$ \\
\hline \multirow[t]{2}{*}{ Fiwa } & Permethrin & Tube & Alive & 5 & 42 & 30 & $\begin{array}{l}\text { Genotypic } \\
p=0.63\end{array}$ \\
\hline & $\mathrm{n}=161$ & & Dead & 8 & 49 & 27 & $\begin{array}{l}\text { Allelic } \\
\mathrm{p}=0.42 \\
\mathrm{OR}^{\mathrm{a}} 1.23 \\
(0.76-2.00)\end{array}$ \\
\hline \multirow[t]{2}{*}{ Fiwa } & Deltamethrir & Tube & Alive & 6 & 14 & 18 & $\begin{array}{l}\text { Genotypic } \\
\mathrm{p}=6.21 \times 10^{-3}\end{array}$ \\
\hline & $\mathrm{n}=78$ & & Dead & 7 & 27 & 6 & $\begin{array}{l}\text { Allelic } \\
\mathrm{p}=0.04 \\
\text { OR } 2.01 \\
(1.01-4.06)\end{array}$ \\
\hline Bassa & Permethrin & Tube & Alive & 1 & 19 & 18 & $\begin{array}{l}\text { Genotypic } \\
\mathrm{p}=0.79\end{array}$ \\
\hline
\end{tabular}




\begin{tabular}{|c|c|c|c|c|c|c|c|}
\hline Site & Insecticide & Bioassay & Phenotype & $\begin{array}{l}\text { Wildtype } \\
\text { homozygote }\end{array}$ & Heterozygote & $\begin{array}{l}\text { Triple } \\
\text { mutant } \\
\text { homozygote }\end{array}$ & $\begin{array}{l}\text { Test and } p \\
\text { value }\end{array}$ \\
\hline & $\mathrm{n}=95$ & & Dead & 2 & 32 & 23 & $\begin{array}{l}\text { Allelic } \\
\mathrm{p}=0.63 ; \\
\text { OR 1.21 } \\
(0.61-2.43)\end{array}$ \\
\hline \multirow[t]{2}{*}{ Bassa } & \multicolumn{2}{|c|}{ Deltamethrin Tube } & Alive & 0 & 3 & 16 & $\begin{array}{l}\text { Genotypic } \\
p=4.02 \times 10^{-3}\end{array}$ \\
\hline & \multicolumn{2}{|l|}{$\mathrm{n}=47$} & Dead & 1 & 16 & 11 & $\begin{array}{l}\text { Allelic } \\
\mathrm{p}=5.71 \times 10^{-3} ; \\
\text { OR } 5.44 \\
(1.41- \\
31.28)\end{array}$ \\
\hline \multirow[t]{2}{*}{ Pwamba } & \multicolumn{2}{|c|}{ Deltamethrin Tube } & Alive & 7 & 9 & 7 & $\begin{array}{l}\text { Genotypic } \\
p=0.066\end{array}$ \\
\hline & \multicolumn{2}{|l|}{$\mathrm{n}=40$} & Dead & 9 & 9 & 1 & $\begin{array}{l}\text { Allelic } \\
\mathrm{p}=0.02 \\
\text { OR } 2.93 \\
(1.07-8.39)\end{array}$ \\
\hline \multirow[t]{2}{*}{ Fiwa } & \multirow{2}{*}{$\begin{array}{l}\text { Olyset } \\
\text { Plus } \\
\mathrm{n}=106\end{array}$} & \multirow[t]{2}{*}{ Net } & Alive & 6 & 21 & 30 & $\begin{array}{l}\text { Genotypic } \\
\mathrm{p}=0.82\end{array}$ \\
\hline & & & Dead & 6 & 15 & 28 & $\begin{array}{l}\text { Allelic } \\
\mathrm{p}=0.88 \\
\text { OR } 0.93 \\
(0.49-1.77)\end{array}$ \\
\hline \multirow[t]{2}{*}{ Fiwa } & \multirow{2}{*}{$\begin{array}{l}\text { Permanet } \\
3.0 \\
n=155\end{array}$} & \multirow[t]{2}{*}{ Net } & Alive & 5 & 26 & 24 & $\begin{array}{l}\text { Genotypic } \\
\mathrm{p}=5.61 \times 10^{-4}\end{array}$ \\
\hline & & & Dead & 30 & 51 & 19 & $\begin{array}{l}\text { Allelic } \\
\mathrm{p}=1.4 \times 10^{-4} \\
\text { OR } 2.56 \\
(1.54-4.31)\end{array}$ \\
\hline
\end{tabular}

\section{References}

1. Ranson, H. and N. Lissenden, Insecticide resistance in African Anopheles mosquitoes: a worsening situation that needs urgent action to maintain malaria control. . Trends in Parasitology, 2016.32 (3): p. 187-196.

2. WHO, World malaria report 2019 , W.H. Organization, Editor. 2019, World Health Organization: Geneva. p. 232.

3. Kleinschmidt, I., et al., Implications of insecticide resistance for malaria vector control with long-lasting insecticidal nets: a WHO-coordinated, prospective, international, observational cohort study. Lancet Infectious Diseases, 2018. 18 (6): p. 640-649.

4. Staedke, S.G., Gonahasa, S., Dorsey, G, Kamya, M.R., Maiteki-Sebuguzi, C., Lynd, A., Katureebe, A., Kyohere, M., Mutungi, P., Kigozi, S.P., Opigo, J., Hemingway, J., and M.J. Donnelly, Effect of longlasting insecticidal nets with and without piperonyl butoxide on malaria indicators in Uganda (LLINEUP): a pragmatic, cluster-randomised trial embedded in a national LLIN distribution campaign. Lancet, 2020(395): p. $1292-303$. 
5. Killeen, G.F. and H. Ranson, Insecticide-resistant malaria vectors must be tackled. Lancet, 2018.391 (10130): p. 1551-1552.

6. The Anopheles gambiae 1000 Genomes Consortium, Genome variation and population structure among 1142 mosquitoes of the African malaria vector species Anopheles gambiae and Anopheles coluzzii. Genome Research, 2020. 30 (10): p. 1533-1546.

7. Mugenzi, L.M.J., et al., Cis-regulatory CYP6P9b P450 variants associated with loss of insecticide-treated bed net efficacy against Anopheles funestus. Nature Communications, 2019. 10 .

8. Donnelly, M.J., A. Isaacs, and D. Weetman, Identification, validation, and application of molecular diagnostics for insecticide resistance in malaria vectors. Trends in Parasitology, 2016.32 (3): p. 197-206.

9. Mitchell, S.N., et al., Metabolic and Target-Site Mechanisms Combine to Confer Strong DDT Resistance in Anopheles gambiae. PLoS ONE, 2014. 9 (3).

10. Weetman, D., Wilding, C.S., Neafsey, D.E., Muller, P., Ochomo, E., Isaacs, A.T., Steen, K., Rippon, E.J., Morgan, J.C., Mawejee, H.D., Rigden, D.J., Okedi, L.M., Donnelly, M.J. , Candidate-gene based GWAS identifies reproducible DNA markers for metabolic pyrethroid resistance from standing genetic variation in East African Anopheles gambiae. Scientific Reports, 2018. 8 : p. e2920.

11. Weetman, D. and M.J. Donnelly, Evolution of insecticide resistance diagnostics in malaria vectors. Transactions of The Royal Society of Tropical Medicine and Hygiene, 2015. 109 (5): p. 291-293.

12. Bhatt, S., et al., The effect of malaria control on Plasmodium falciparum in Africa between 2000 and 2015. Nature, 2015.526 (7572): p. 207-+.

13. Hancock, P.A., et al., Mapping trends in insecticide resistance phenotypes in African malaria vectors. PLoS Biology, 2020.18 (6): p. e3000633.

14. Clarkson, C.S., et al., The genetic architecture of target-site resistance to pyrethroid insecticides in the African malaria vectors Anopheles gambiae and Anopheles coluzzii. bioRxiv, 2018(https://doi.org/10.1101/323980).

15. Müller, P., et al., Field-caught permethrin-resistant Anopheles gambiae overexpress CYP6P3, a P450 that metabolises pyrethroids. PLoS Genetics, 2008. 4 (11): p. e1000286.

16. Weedall, G.D., et al., A cytochrome P450 allele confers pyrethroid resistance on a major African malaria vector, reducing insecticide-treated bednet efficacy. Science Translational Medicine, 2019.11 (484).

17. Protopopoff, N., et al., Effectiveness of a long-lasting piperonyl butoxide-treated insecticidal net and indoor residual spray interventions, separately and together, against malaria transmitted by pyrethroid-resistant mosquitoes: a cluster, randomised controlled, two-by-two factorial design trial. Lancet, 2018.391 (10130): p. 1577-1588.

18. Lynd, A., et al., LLIN Evaluation in Uganda Project (LLINEUP) - A cross-sectional survey of species diversity and insecticide resistance in 48 districts of Uganda. Parasites and Vectors, 2019.12 : p. e94.

19. The Anopheles gambiae 1000 Genomes Consortium, Natural diversity of the malaria vector Anopheles gambiae. Nature (London), 2017. 552 : p. 96-100.

20. Lucas, E., et al., Whole genome sequencing reveals high complexity of copy number variation at insecticide resistance loci in malaria mosquitoes. Genome Research, 2019. https://doi.org/10.1101/399568 (29): p. 1250-1261

21. Ibrahim, S.S., et al., Pyrethroid resistance in the major malaria vector Anopheles funestus is exacerbated by overexpression and overactivity of the P450 CYP6AA1 across Africa. Genes, 2018.9 (3): p. 17.

22. Zhou, D., et al., Genomic analysis of detoxification supergene families in the mosquito Anopheles sinensis. PLoS One, 2015.10 (11): p. e0143387. 
23. Kwiatkowska, R.M., et al., Dissecting the mechanisms responsible for the multiple insecticide resistance phenotype in Anopheles gambiae s.s., M form, from Vallee du Kou, Burkina Faso. Gene, 2013. 519 (1): p. 98-106.

24. Mitchell, S., et al., Identification and validation of a gene causing cross-resistance between insecticide classes in Anopheles gambiae from Ghana. Proceedings of the National Academy of Sciences of the United States of America, 2012. 109 : p. 6147-6152

25. Edi, C.V., et al., CYP6 P450 enzymes and ACE-1 duplication produce extreme and multiple insecticide resistance in the malaria mosquito Anopheles gambiae. PLoS Genetics, 2014. 10 (3): p. e1004236-e1004236.

26. Vontas, J., et al., Rapid selection of a pyrethroid metabolic enzyme CYP9K1 by operational malaria control activities. Proceedings of the National Academy of Sciences of the United States of America, 2018.115 (18): p. 4619-4624.

27. Miles, A., et al., cggh/scikit-allel . 2019, Zenodo.

28. Garud, N.R., et al., Recent selective sweeps in North American Drosophila melanogaster show signatures of soft Sweeps. PLOS Genetics, 2015. 11 (2): p. e1005004.

29. Wat'senga, F., et al., Intensity of pyrethroid resistance in Anopheles gambiae before and after a mass distribution of insecticide-treated nets in Kinshasa and in 11 provinces of the Democratic Republic of Congo. Malaria Journal, 2020. 19 (1): p. e169.

30. Lynd, A., et al., Insecticide resistance in Anopheles gambiae from the northern Democratic Republic of Congo, with extreme knockdown resistance ( $k d r)$ mutation frequencies revealed by a new diagnostic assay. Malaria Journal, 2018. 17 .

31. Lynd, A., Weetman, D., Barbosa, S., Yawson, A.E., Mitchell, S., Pinto, J., Hastings, I. and Donnelly, M.J., Field, genetic and modelling approaches show strong positive selection acting upon an insecticide resistance mutation in Anopheles gambiae s.s. Molecular Biology and Evolution, 2010. 27 : p. 1117-1125.

32. Yunta, C., et al., Cross-resistance profiles of malaria mosquito P450s associated with pyrethroid resistance against WHO insecticides. Pesticide Biochemistry and Physiology, 2019.161 : p. 61-67.

33. Yeka, A., et al., Malaria in Uganda: Challenges to control on the long road to elimination I. Epidemiology and current control efforts. Acta Tropica, 2012. 121 (3): p. 184-195.

34. Ministry of Health, R.o.K., Insecticide Resistance Management Plan, Kenya 2020 - 2024 . 2019, Ministry of Health, Republic of Kenya.

35. Ruiz, J.L., L.C. Ranford-Cartwright, and E. Gomez-Diaz, The regulatory genome of the malaria vector Anopheles gambiae: integrating chromatin accessibility and gene expression. bioRxiv, 2020: $p$. 2020.06.22.164228.

36. Liu, B., et al., iEnhancer-2L: a two-layer predictor for identifying enhancers and their strength by pseudo k-tuple nucleotide composition. Bioinformatics, 2015. 32 (3): p. 362-369.

37. Liu, B., et al., iEnhancer-EL: identifying enhancers and their strength with ensemble learning approach. Bioinformatics, 2018.34 (22): p. 3835-3842.

38. Schmidt, J.M., et al., Copy number variation and transposable elements feature in recent, ongoing adaptation at the Cyp6g1 locus.PLoS Genetics, 2010. 6 (6): p. e1000998.

39. Jones, C., et al., Footprints of positive selection associated with a novel mutation (N1575Y) in the voltage gated sodium channel of Anopheles gambiae. Proceedings of the National Academy of Sciences of the United States of America, 2012. 109 : p. 6614-6619.

40. Djogbenou, L., et al., Ace-I duplication in Anopheles gambiae: a challenge for malaria control. Malaria Journal, 2009. 8 . 
41. Boakye, D.A., et al., Patterns of household insecticide use and pyrethroid resistance in Anopheles gambiae sensu stricto (Diptera: Culicidae) within the Accra metropolis of Ghana. African Entomology, 2009. 17 (2): p. $125-130$.

42. Grau-Bove, X., et al., Evolution of the insecticide target Rdl in African Anopheles is driven by interspecific and interkaryotypic introgression. bioRxiv, 2020: p. 2019.12.17.879775.

43. Neafsey, D.E., et al., Highly evolvable malaria vectors: The genomes of 16 Anopheles mosquitoes. Science, 2015. 347 (6217): p. 43-+.

44. Nene, V., et al., Genome sequence of Aedes aegypti, a major arbovirus vector. Science, 2007. 316 (5832): p. $1718-1723$.

45. Buss, D.S. and A. Callaghan, Molecular comparisons of the Culex pipiens (L.) complex esterase gene amplicons. Insect Biochemistry and Molecular Biology, 2004. 34 (5): p. 433-441.

46. Rugnao, S., et al., LLIN Evaluation in Uganda Project (LLINEUP): factors associated with childhood parasitaemia and anaemia 3years after a national long-lasting insecticidal net distribution campaign: a crosssectional survey. Malaria Journal, 2019. 18 : p. e207.

47. Newcombe, R.G., Two-sided confidence intervals for the single proportion: comparison of seven methods. Statistics in Medicine, 1998.17 : p. 857-872. 